\title{
Neutrino Nucleus Deep Inelastic Scattering at MINERvA
}

\author{
Marianette Wospakrik* \\ University of Florida \\ E-mail: wospakrkefnal.gov
}

\begin{abstract}
Neutrino-nucleus charged-current deep inelastic scattering (DIS) provides a complementary probe to charged lepton-nucleus DIS in the study of nuclear and hadronic structure. The MINERvA experiment is a dedicated neutrino scattering experiment located on the NuMI beamline in Fermilab. With multiple nuclear targets of $\mathrm{Pb}, \mathrm{Fe}, \mathrm{CH}$, and $\mathrm{C}$ in the same beam, MINERvA has the capability to add to the world knowledge of DIS that still contains poorly understood nuclear effects. The recent change of the NuMI beam line to the Medium Energy configuration has increased both the intensity and average neutrino energy thereby greatly improving the projected sensitivities for nuclear and hadronic structure analyses utilizing MINERvA's multiple nuclear targets. The measurement of the ratio on $\mathrm{C}, \mathrm{Fe}, \mathrm{Pb}$ to $\mathrm{CH}$ using the MINERvA low energy data set will be discussed as well as the current state of DIS analysis with the medium energy data set.
\end{abstract}

XXV International Workshop on Deep-Inelastic Scattering and Related Subjects

3-7 April 2017

University of Birmingham, $U K$

\footnotetext{
*Speaker.
} 


\section{Introduction}

Charged-lepton deep inelastic scattering (DIS) has been used as a parton-level tool for exploring nuclear effects on a variety of targets. These partonic nuclear effects are usually described as a function of four-momentum transfer squared $Q^{2}=q^{2}$ and the Bjorken scaling variable $x_{B j}$, the fraction of the nucleon's momentum carried by the struck parton in the infinite momentum frame:

$$
x_{B j}=\frac{Q^{2}}{2 m_{n} E_{H A D}}
$$

where $m_{n}$ is the average of proton and neutron masses and $E_{H A D}$ is the total energy of the final state hadrons. Charged-lepton DIS data yielded a wealth of information on partonic nuclear structure and interaction mechanisms that leads to the characterization of four distinct partonic nuclear effects in different kinematical regions as function of the $x_{B j}$ variable: shadowing effect at $x_{B j} \lesssim 0.1$, anti-shadowing effect $0.1 \lesssim x_{B j} \lesssim 0.3$, EMC effect $0.3 \lesssim x_{B j} \lesssim 0.75$, and Fermi motion effect $x_{B j} \gtrsim 0.75$.

The neutrino DIS cross section data is a complementary tool to charged lepton-nucleus DIS for probing the nuclear and hadronic structure. Neutrinos are uniquely sensitive to both the axial vector and vector components, and therefore the nuclear dependence observed in neutrino and charged-lepton DIS is expected to be different.The existing neutrino DIS measurements are done in heavy nuclei such as $\mathrm{Fe}$, and $\mathrm{Pb}$ ([1],[2]) and comparing these measurements to free-nucleon to determine neutrino-nuclear effects has generated some tension with charged-lepton partonic nuclear effects [3]. Despite these unresolved inconsistencies, the existing charged lepton nuclear effects are adapted directly into neutrino event generators, such as GENIE.

\section{The MINERvA Detector}

The MINERvA experiment (Main INjector ExpeRiment for $v$-A) is a neutrino scattering experiment located at Fermi National Accelerator Laboratory which is designed to precisely measure the cross sections of different neutrino interaction. It utilizes the intense NuMI (Neutrinos at Main Injector) beam, which is produced when $120 \mathrm{GeV}$ protons hit the carbon target. The MINERvA detector consists of 120 horizontally stacked modules and is divided into four regions: the nuclear target region, the active tracker region, electromagnetic calorimetry, and hadronic calorimetry [4]. A schematic view of the MINERvA detector with the different sub-detectors labeled is in Fig. 1. MINERvA employs nuclear targets consisting of of helium, carbon, iron, lead, and water placed upstream of the finely-segmented scintillator-based tracking region, in order to measure how neutrino interactions differ depending on what kind of atomic nucleus is involved in the interaction. The various nuclear targets are separated from each other by four active tracker modules to allow good vertexing resolution for events originating in the nuclear target. Located just downstream of MINERvA is the MINOS near-detector, which serves as a magnetized iron spectrometer to measure the charge and energy of forward-going muons. A schematic of the nuclear target region of MINERvA is shown in Fig. 2. 

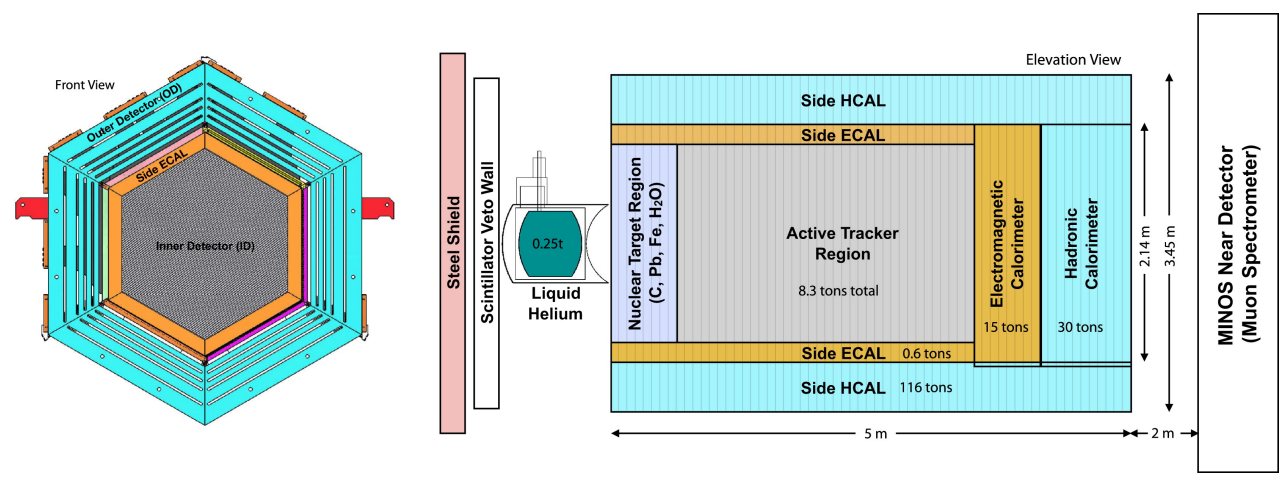

Figure 1: Schematic of the MINERvA detector, with all relevant sub detectors labeled.

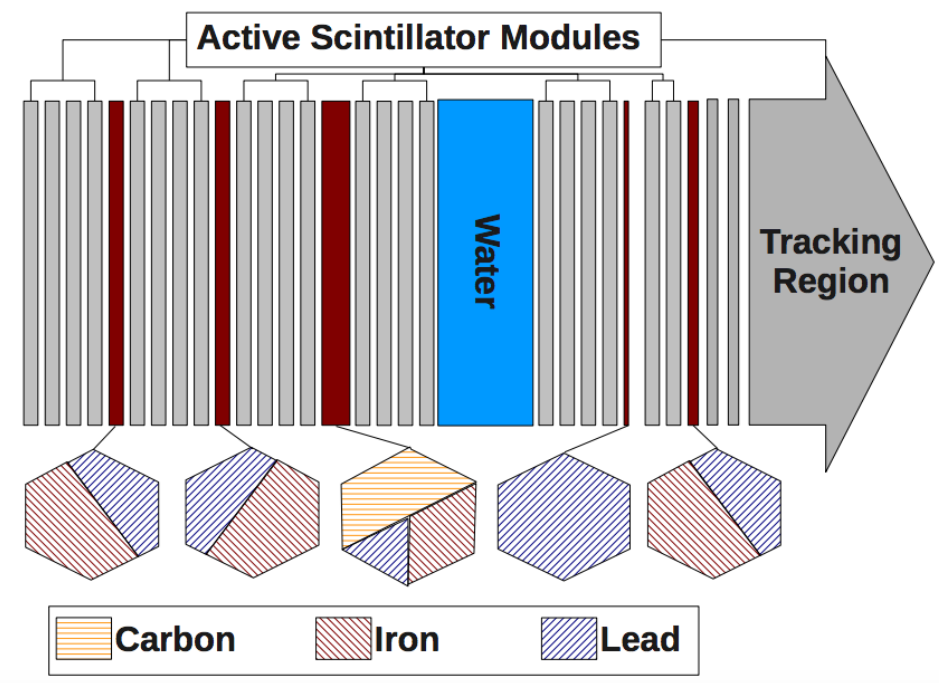

Figure 2: Schematic of the nuclear targets of MINERvA. The tracking region of the MINERvA detector is immediatelydownstream (right) of the nuclear target region.

\section{Event Reconstruction and Analysis}

Identifying charged-current (CC) inclusive interaction is the first step to DIS analysis. In this interaction, we require the charged-current $v_{\mu}$ events to have only one reconstructed $\mu^{-}$in the final state that is matched to the MINOS detector. The requirement that a track is matched in MINOS limits the muon scattering angle with respect to the beam $\left(\theta_{\mu}\right)$ to be less than $17^{\circ}$. Additional deposits of energy in the detector not associated with the muon track and within a -20 to +30 ns window of the event vertex are summed to form a recoil energy. This recoil energy is then calorimetrically corrected for containment and neutral particle content and is defined as the $E_{H A D}$ of the neutrino event. The most upstream cluster of the muon track is used as an anchor for the reconstruction of the vertex. From the vertex $z$ position, we can determine which passive target the interaction coming from and using the vertex $x, y$ position, we can determine in which material $(\mathrm{C}$, $\mathrm{Fe}$ or $\mathrm{Pb}$ ) the interaction originated. The DIS event is then isolated by using the kinematics cut on 
$Q^{2}$ and $W$ defined as:

$$
\begin{gathered}
Q^{2}=4 E_{v} E_{\mu} \sin ^{2}\left(\frac{\theta_{\mu}}{2}\right) \\
W=\sqrt{m_{n}^{2}+\left(2 m_{n}^{2}\left(E_{v}-E_{\mu}\right)\right)-Q^{2}}
\end{gathered}
$$

where the neutrino energy is reconstructed by summing the muon and hadronic energy, $E_{v}=E_{\mu}+$ $E_{H A D}$. An event is considered a DIS event if $Q^{2} \geq 1.0 \mathrm{GeV}^{2} / c^{2}$ and $W \geq 2.0 \mathrm{GeV} / \mathrm{c}$.

\section{Results at $3.5 \mathrm{GeV}$ Beam}

After subtracting backgrounds, the events are corrected for detector acceptance and smearing effects to form a cross-section. Because all targets in MINERvA are exposed to an identical neutrino beam, uncertainties due to the flux simulation largely cancels in these ratios. Fig. 3 shows the ratios of the differential cross-section with respect to $x_{B j}\left(\frac{d \sigma}{d x}\right)$ of $\mathrm{C}, \mathrm{Fe}$ and $\mathrm{Pb}$ to $\mathrm{CH}$. A smaller than expected ratio is observed in the lowest $\mathrm{x}$-bin $\left(0.0<x_{B j}<0.1\right)$ for the ratio of $\mathrm{Pb}$ to $\mathrm{CH}$ which suggests additional nuclear shadowing in $\mathrm{Pb}$ versus $\mathrm{CH}$. Other bins of Bjorken-x shows good agreement between the data and prediction especially in the largest $x_{B j}$ bin, $0.4<x_{B j}<0.75$, which is dominated by the EMC effect [5]. The data collected with the $3.5 \mathrm{GeV}$ beams lack the statistical precision to differentiate between different explanations of the EMC effect. Future studies with MINERvA will use a higher average energy neutrino spectrum, and will probe this interesting shadowing region by decreasing the average $x_{B j}$ of neutrino DIS events.

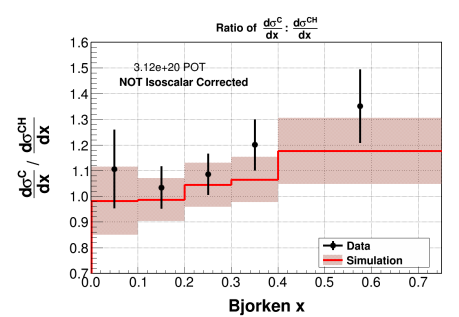

(a) $\mathrm{C} / \mathrm{CH}$

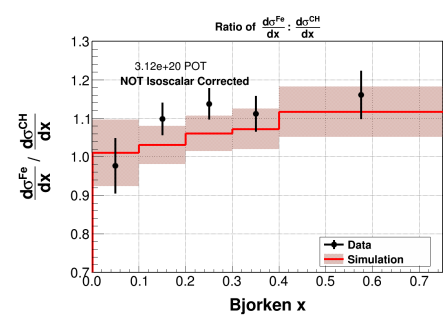

(b) $\mathrm{Fe} / \mathrm{CH}$

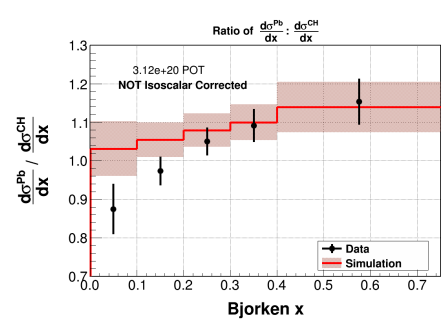

(c) $\mathrm{Pb} / \mathrm{CH}$

Figure 3: Ratio of the $\mathrm{x}$-differential DIS cross section on $\mathrm{C}, \mathrm{Fe}$, and $\mathrm{Pb}$ to $\mathrm{CH}$. Data are drawn as black points with the statistical uncertainty drawn as the error bar. The red line is the prediction, and the band surrounding the prediction is the $1 \sigma$ systematic uncertainties. The total systematic error is drawn as a band around the simulation in each histogram.

\section{Improvement at $6 \mathrm{GeV}$ Beam}

MINERvA is currently taking data in the increased beam energy mode which peaks at $\sim 6$ $\mathrm{GeV}$. We have taken $\sim 12 \times 10^{20}$ Protons on Target (POT) in neutrino mode and currently taking data in anti-neutrino mode. This is about three times more POT than for the data reported in Sect. 4 , and has a more focused beam and twice the average cross section. This higher statistics yields will improve comparisons across nuclei and, as the peak of energy now moves to the DIS-rich kinematic region, better probes the nuclear structure functions. 
It is important to precisely determine the neutrino interaction vertex because vertexing is the primary tool to identify the target nucleus required to study $A$-dependent nuclear effects. The traditional vertex reconstruction methods rely only on tracking information and for events with high hadronic energy such as DIS, this method can fail when the shower activity obscures the vertex location or when tracks created by secondary interactions or decays bias the vertex location. In the target analysis, missing the vertex by a few centimeters may pose a significant problem.

However, with the increased beam energy, there is an increase of events with misreconstructed vertices and the general strategy of rejecting events with these misreconstructed vertices may reduce the statistics gained. Events in MINERvA can be represented as images and the vertex finding can be represented as an image classification problem that is well suited for a machine learning application. In particular, we utilize the deep convolutional neural networks to localize neutrino interaction vertices in the MINERvA nuclear target regions.

Neural networks roughly model operation of neurons in the human brain. The nodes of the input and output layers are connected by weighted connections. Neural networks become "deep" when the outputs of one network become inputs of another, creating many hidden layers in the network. Deep Convolutional Neural Networks (DCNN) ([6] [7], [8]) are a type of deep neural networks, where the inputs of each layer are mapped into outputs by convolving a kernel with the inputs. By using the same weights for the kernel across the input, the network is able to discover translationally invariant features. Stacking multiple layers with many kernels per layer allows a DCNN to automatically extract features that describe the input image. In the DCNN application, we are effectively treating localization as a classification problem. We label the nuclear target region as 11 segments, where the odd segments refer to the passive target and the even segments refer to the scintillator trackers between the targets as well as the regions upstream and downstream of the nuclear target region. We then ask the DCNN to predict which segment out of the 11 segments the neutrino interaction vertices located. Fig. 4 shows the performance comparisons between trackbased vertexing and DCNN vertexing as row-normalized confusion matrices (the sum of the matrix entries reading along a row are normalized to one) and the diagonal elements can be interpreted as the number of segments correctly defined as a fraction of true vertices originating in the segment. The linear color scale shows comparable performance between track-based reconstruction and DCNN performance, but the $\log _{10}$ (percentages) scale shows the improved performance from DNN reconstruction. Less smearing from the diagonal elements is observed in the DCNN reconstruction compared to track-based indicating better purity of the event samples which will lead to better statistical precision.

\section{Conclusion}

We have seen an improvement in vertexing using DCNN reconstruction in comparison with the track-based reconstruction for the $6 \mathrm{GeV}$ neutrino energy beam, which will optimize the DIS event selection required to probe the kinematical regions, in particular for the shadowing region where a depletion is observed in the cross section of $\mathrm{Pb}$ to $\mathrm{CH}$, at smaller average Bjorken-x. MINERvA has finished taking data in the neutrino mode and is currently taking data in the anti-neutrino mode which will allow us to do further measurements of structure function $F_{3}$ by looking at the difference between neutrino and anti-neutrino DIS cross sections. 

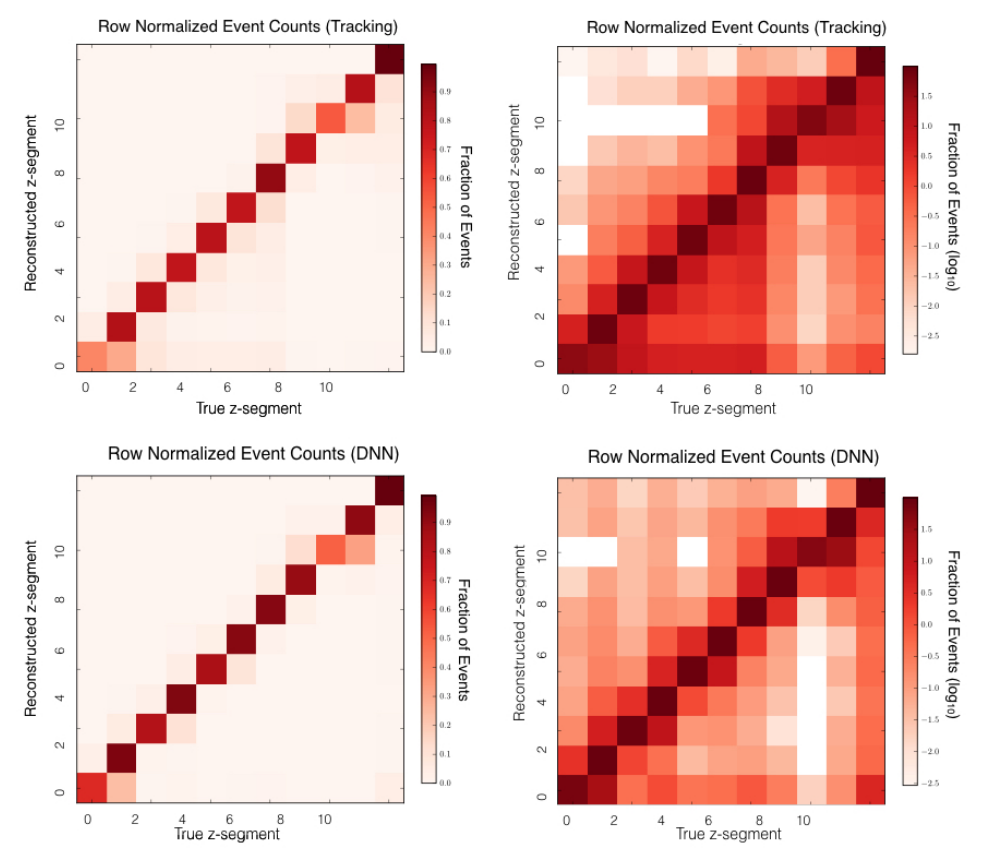

Figure 4: Track-based reconstruction performance (top row) and deep neural network reconstruction performance (bottom row) as row-normalized confusion matrices. The plots on the left have a linear color scale, and the plots on the right show $\log _{10}$ (percentages).

\section{References}

[1] B. T. Fleming et al. (CCFR/NuTeV Collaboration), "First Measurement of the Low $x$, Low $Q^{2}$ Structure Function $F_{2}$ in Neutrino Scattering," Phys. Rev. Lett. 86, 5430 (2001).

[2] G. Onengut , et al. (CHORUS Collaboration), "Measurement of nucleon structure functions in neutrino scattering," Phys. Lett. B 632, 65 (2006).

[3] I. Schienbein, et al., "Parton distribution function nuclear corrections for charged lepton and neutrino deep inelastic scattering processes," Phys. Rev. D 80.094004 (2009)

[4] L. Aliaga, et al. [MINERvA Collaboration], "Design, Calibration, and Performance of the MINERvA Detector," Nucl.Instrum.Meth. A743 130-159 (2014).

[5] J. Mousseau, et al. [MINERvA Collaboration], "Measurement of partonic nuclear effects in deep-inelastic neutrino scattering using MINERvA," Phys. Rev. D 93, 071101(2016).

[6] Y. LeCun, L.D. Jackel, L. Bottou, A. Brunot, C. Cortes, J.S. Denker, H. Drucker, I. Guyon, U.A., E. Sackinger, et al., "Comparison of learning algorithms for handwritten digit recognition", International Conference on Artificial Neural Networks 60, 53-60 (1995).

[7] A. S. Razavian, H. Azizpour, J. Sullivan, S. Carlsson, "CNN Features off-the-shelf: an Astounding Baseline for Recognition", arXiv:1403.6382 (2014).

[8] A. Aurisano, A. Radovic, D. Rocco, A. Himmel, M. D. Messier, E. Niner, G. Pawloski, F. Psihas, A. Sousa, P. Vahle, "A Convolutional Neural Network Neutrino Event Classifier", JINST 11, P09001 (2016). 